\title{
Assessment of pulmonary valve and right ventricular outflow tract with real-time three-dimensional echocardiography
}

\author{
Ashraf M. Anwar • Osama Soliman · Annemien E. van den Bosch • \\ Jackie S. McGhie · Marcel L. Geleijnse · Folkert J. ten Cate • \\ Folkert J. Meijboom
}

Received: 21 June 2006/ Accepted: 24 July 2006/Published online: 8 September 2006

(C) Springer Science+Business Media B.V. 2006

\begin{abstract}
Aim Assessment of pulmonary valve (PV) and right ventricular outflow tract (RVOT) using realtime 3-dimensional echocardiography (RT3DE). Methods Two-dimensional echocardiography (2DE) and RT3DE were performed in 50 patients with congenital heart disease (mean age $32 \pm 9.5$ years, $60 \%$ female). Measurements were obtained at parasternal views: short axis (PSAX) at aortic valve level and long axis (PLAX) with superior tilting. RT3DE visualization was evaluated by 4-point score (1: not visualized, 2 : inadequate, 3: sufficient, and 4: excellent). Diameters of PV annulus (PVAD), and RVOT (RVOTD) were measured by both 2DE and RT3DE, while areas (PVAA) and (RVOTA) by RT3DE only. Results By RT3DE, PV was visualized sufficiently in $68 \%$ and RVOT excellently in $40 \%$. PVAD and PVAA were measured in $88 \%$. RVOTD and PVAD by 2DE at PLAX were significantly higher than PSAX $(P<0.0001)$ and lower than that by RT3DE $(P<0.001)$.
\end{abstract}

A. E. van den Bosch · J. S. McGhie .

M. L. Geleijnse · F. J. ten Cate · F. J. Meijboom ( $₫)$ Department of Cardiology, Thoraxcenter, Erasmus MC, Room Ba 302, Dr. Molewaterplein 40, 3015 GD Rotterdam, The Netherlands

e-mail: f.j.meijboom@erasmusmc.nl

A. M. Anwar · O. Soliman

Department of Cardiology, Al-Husein University

hospital, Al-Azhar University, Cairo, Egypt
Conclusion RT3DE helps in RVOT and PV assessment adding more details supplemental to 2DE.

Keywords Real time 3-dimensional echocardiography (RT3DE) - Right ventricular outflow tract $(\mathrm{RVOT}) \cdot$ Pulmonary valve $(\mathrm{PV})$

\section{Introduction}

The right ventricular outflow tract (RVOT) extends from the antero-superior aspect of the right ventricle to the pulmonary artery (PA), and includes the pulmonary valve (PV). It is best imaged by 2-dimensional echocardiography (2DE) from the parasternal long axis view angulated superiorly and the parasternal short axis at the base of the heart. It can also be imaged from the subcostal long and transverse windows and the apical window [1]. Visualization of the PV leaflets by $2 \mathrm{DE}$ in long axis is limited, especially in adults, because 2DE images only one or two leaflets well and a short axis view often is not obtainable [2]. The level of RVOT obstruction can be determined using pulsed Doppler and color flow to identify the anatomic site at which the flow velocity increases and the post-stenotic flow disturbance appears. The obstruction itself may be depicted on 2DE as a muscular subpulmonic ridge, as deformed 
doming PV valve leaflet or as narrowing in the PA [2]. Transesophageal echocardiography helps in imaging RVOT from a high esophageal position at $0^{\circ}$ rotation with a long axis view of the PA from the valve plane to its bifurcation. The PV also may be visualized in the $90^{\circ}$ long-axis plane. The PV is seen in its perpendicular relationship to the aortic valve in the far field of the image, so it remains virtually impossible to assess the precise morphology of valve cusps or its commissures [3]. Magnetic resonance imaging may add to the assessment of the obstruction level, PA size and may detect other associated lesions such as PA stenosis and coexisting pulmonary regurgitation [4]. Until now, it has not been possible to visualize all three leaflets of the PV simultaneously by non-invasive diagnostic tools except in rare cases in which the valve pushed interiorly [5]. The introduction of 3dimensional echocardiography (3DE) helps in displaying the intracardiac anatomy in views that are similar to the ones encountered during operation [6]. With more advances in the techniques, probe and software, it is now possible to assess intracardiac structure and function accurately within a reasonable time with the Real time 3DE (RT3DE). We hypothesize that RT3DE will clarify the knowledge of PV anatomy and RVOT by direct visualization from all aspects. The study aimed to use RT3DE in assessment of the morphology of pulmonary valve (PV) and right ventricular outflow tract (RVOT) and comparing its measurements with that obtained by $2 \mathrm{DE}$.

\section{Subjects and methods}

Fifty patients (mean age $32 \pm 9.5$ years, $60 \%$ female) (Table 1) referred to the echocardiography lab for evaluation of adult congenital heart disease were included. All patients were in sinus rhythm. In all 2DE was done with the patient in the left lateral decubitus position using both apical and parasternal views with a commercially available ultrasound system (Philips Sonos 7500 with $3.5 \mathrm{MHz}$ probe, Best, The Netherlands). RVOT and PV were examined at parasternal short axis (PSAX) view at aortic valve level and
Table 1 Distribution of congenital heart defects in studied patients

\begin{tabular}{ll}
\hline Type of congenital defect & $\begin{array}{l}\text { Number of } \\
\text { patients (\%) }\end{array}$ \\
\hline Atrio-ventricular septal defect (AVSD) & $13(26 \%)$ \\
Valvular Pulmonary stenosis (PS) & $7(14 \%)$ \\
Post defect closure & $7(14 \%)$ \\
Atrial septal defect (ASD) & $5(10 \%)$ \\
Atrial septal defect + & $4(8 \%)$ \\
$\quad$ Ventricular septal defect & \\
Transposed great arteries (TGA) & $4(8 \%)$ \\
Ventricular septal defect (VSD) & $3(6 \%)$ \\
Ebstein's anomaly & $2(4 \%)$ \\
Coronary AV fistula & $2(4 \%)$ \\
Normal & $2(4 \%)$ \\
Fallot's tetralogy & $1(2 \%)$ \\
\hline
\end{tabular}

parasternal long axis (PLAX) with moving transducer towards the base angulating superiorly. The subtotal short axis view at aortic valve level was used instead of parasternal one in 10 cases. Zooming was used to clarify images and minimize artefacts. The following variables were measured in each patient [7]: (1) RVOT diameter (RVOTD) measured just proximal to the PV at both views at its maximal width (end-diastole), (2) PV annulus diameter (PVAD) at same frame, (3) proximal PA diameters (PPAD), and (4) distal PA diameters (DPAD) just before its bifurcation. Measurements were obtained only from good image quality without echo dropout.

RT3DE was done by the same ultrasound system attached to a X4 matrix array transducer, capable of providing real-time B-mode images. 3D images were collected approximately within 5-7 s of breath holding in full volume mode. The 3D data set was transferred to an offline analysis software package (TomTec, Munich, Germany). Data were stored digitally and subsequently evaluated by two blinded observers (AMA, $\mathrm{JMcG}$ ). Data analysis of 3D echo imaging had been based on a $2 \mathrm{D}$ approach relying on the echo images obtained from both views (PSAX and PLAX). By using the crop function for image formatting, multiple cross-sections through the scanned volume were made. We selected the narrowest slice to enable visualization of RVOT and PV from both aspects (proximal ventricular and distal pulmonic). RT3DE gain and brightness were adjusted to improve delineation of anatomic 
structures. The relevant features of RVOT, PV, PPA and DPA were checked and classified according to a subjective 4-point scale for image quality $(1=$ not visualized, $2=$ inadequate, 3 = sufficient and $4=$ excellent). By manual tracing of the inner border of RVOT and the PVA, the surface area was automatically delineated and could be visualized from different points of view. The following measures were obtained: (1) RVOTD included maximum RVOTD (max RVOTD) defined as the widest diameter of the RVOT, and minimum RVOTD (min RVOTD) defined as the shortest diameter of the RVOT, (2) RVOT area (RVOTA): measured at its maximal width (end-diastole), (3) PVAD: included two diameters: included maximum PVAD (max PVAD) defined as the widest diameter of the PVA, and minimum PVAD (min PVAD) defined as the shortest diameter of the PVA, (4) PVA area (PVAA): measured at its maximal width (end-diastole), and (5) PPAD, DPAD.

\subsection{Statistical analysis}

All data obtained by 2D TTE and RT3DE were presented as mean $\pm \mathrm{SD}$ to determine whether the difference in values between both techniques was statistically significant or not. A paired sample $t$-test was performed for comparing between means of variables. The level of significance was set to $P<0.05$. Interobsever agreement for visualization score was estimated using kappa values for each morphologic feature and classified as excellent with value of $0.93-1.0$, very good $0.81-0.92$, good $0.61-0.80$ and fair $0.41-0.60$ [8]. For 2DE measurements, a random sample consisted of 20 patients was selected and $t$-test was applied for interobserver variability. Interobserver variability for RT3DE measurements in all patients was estimated according to the Bland and Altman method [9].

\section{Results}

\subsection{RVOT and PV by $2 \mathrm{DE}$}

Higher percentage of clear visualization and measurements of RVOT and PVA could be obtained from the PLAX view than from the PSAX view. RVOTD could be measured from the PLAX view in 28 patients $(56 \%)$ and in 26 patients $(52 \%)$ from the PSAX view. PVAD was measured in 48 patients $(96 \%)$ from the PLAX view and in 46 patients (92\%) from the PSAX view. PPAD and DPAD were recorded from both views in equal percentage $(78 \%$ for PPA and $42 \%$ for DPA). Measurements of RVOTD and PVAD from both views correlated well (Fig. 2A-D). However, RVOTD and PVAD were significantly higher as measured from the PLAX view than from the PSAX $(P<0.0001)$.

\subsection{RVOT and PV by RT3DE}

Acquisition and analysis of RT3DE data were performed successfully within 5-7 min per patient. RVOT was visualized excellently in 20 patients $(40 \%)$ and not visualized in 24 patients $(48 \%)$. Its shape appeared as an oval shape and not completely circular (Fig. 1A). Calculated RVOTA, max RVOTD and min RVOTD were obtained in 26 patients $(52 \%)$. The mean RVOTA was $41.5 \pm 33.4 \mathrm{~mm}$. PVA was visualized excellently in 44 patients $(88 \%)$ and was not visualized in two patients (4\%). The mean PVAA was $33.5 \pm 30.1 \mathrm{~mm}$. PV was visualized sufficiently in 34 patients (68\%) and excellently in 12 patients $(24 \%)$. The number, thickness and mobility of cusps could be studied in 35 patients $(70 \%)$. Bicuspid pulmonary valve was seen in three patients. The diagnosis of the three patients was transposed great arteries, Fallot's tetralogy, and pulmonary stenosis. Pulmonary valve commissures and lines of closure were seen in 35 patients (70\%) (Fig. 1C-D). Pulmonary valve area could not be obtained well in a considerable number of patients with high interobserver variability.

\subsection{Comparison of 2DE and RT3DE}

The RT3DE quadscreen showed that RVOTD obtained by 2DE from PSAX view was perpendicular on the max RVOTD and parallel to the min RVOTD, while RVOTD obtained from PLAX was parallel to the max RVOTD but was 


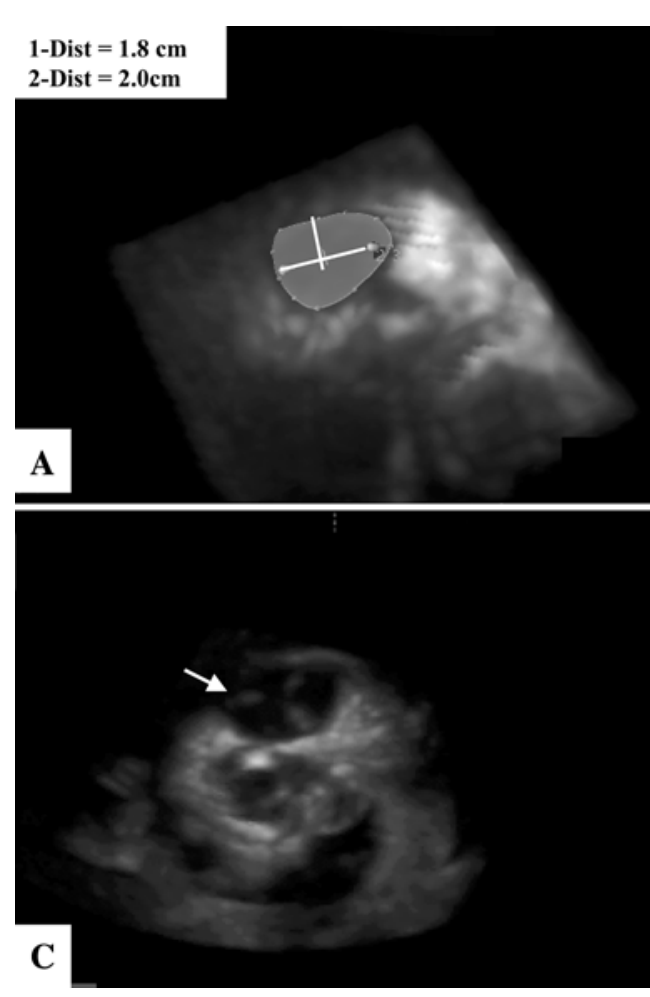

Fig. 1 (A) RT3DE showed the morphology of the traced oval shaped RVOT. The vertical line (1) appears inside it is the RVOTD measured by 2DE at PSAX, and the horizontal line (2) is RVOTD at PLAX. Both lines are away from the true RVOTD, (B) Traced PVA visualized

not extended to the annular ends. The same findings were found for PVAD (Fig. 1A, B). Measurements of RVOTD with 2DE and RT3DE were significantly correlated with each other (Fig. 2B, C), and also for PVAD (Fig. 2E, F). The max RVOTD was significantly larger than RVOTD measured by 2DE from both views $(P<0.0001)$, while the min RVOTD was comparable to RVOT measured from PSAX view $(P=0.8)$. PVAD measurement by RT3DE was significantly larger than that measured by $2 \mathrm{DE}$ from both views $(P<0.0001)$. The value for RT3DE application in comparison with $2 \mathrm{DE}$ is shown in Table 4.

\subsection{PPA and DPA by 2DE and RT3DE}

Measurements of PPAD and DPAD were equal in both 2DE views (PSAX and PLAX). By

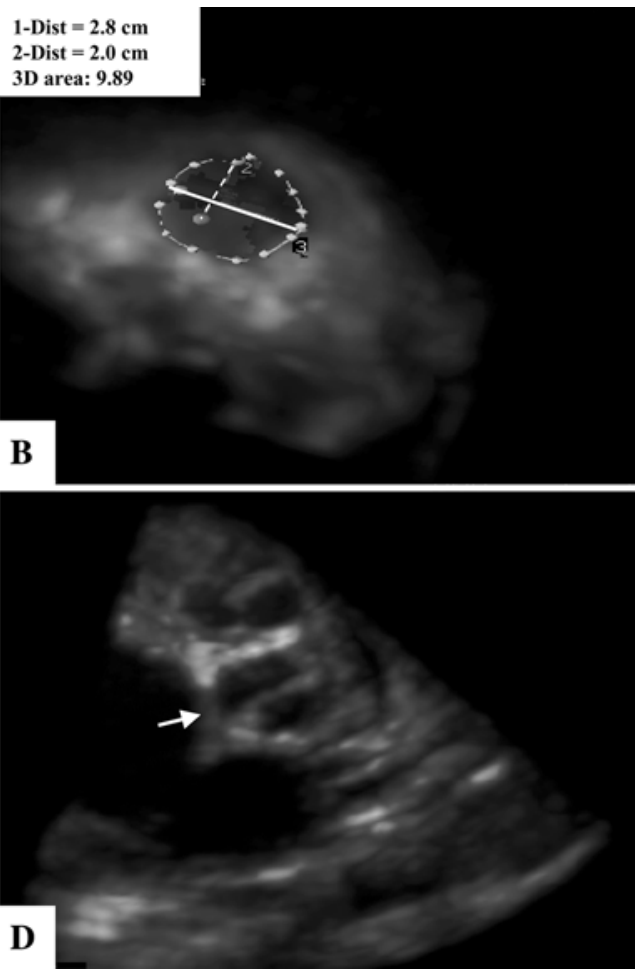

by RT3DE. The horizontal line (1) is PVAD measured by RT3DE and vertical dashed line is PVAD by 2DE in PLAX, (C) RT3DE image of closed tricuspid pulmonary valve with its closure lines and (D) Bicuspid pulmonary valve with central closure line

RT3DE, PPA was excellently visualized in 29 patients $(58 \%)$ and sufficiently in 10 patients $(20 \%)$. DPA was excellently visualized in 15 patients $(30 \%)$ and not visualized in 27 patients (54\%) (see Table 3). Measurements of PPAD and DPAD by 2DE and RT3DE were comparable (see Table 2).

\subsection{Interobsever correlation and agreement}

The agreement for visualization score by RT3DE was fair for the assessment of pulmonary valve (Kappa value: 0.59), good for RVOT (Kappa value: 0.71 ) and very good for PVA, PPA and DPA (Kappa value: 0.91). Excellent interobserver correlation was found for RT3DE measurements $(r=0.94, P<0.0001)$ for RVOTD, and PVAD. Excellent interobserver correlation also was found for 2DE $(r=0.98, \quad P<0.0001)$ 
RVOTD by 2DE at PSAX and PLAX

(A)

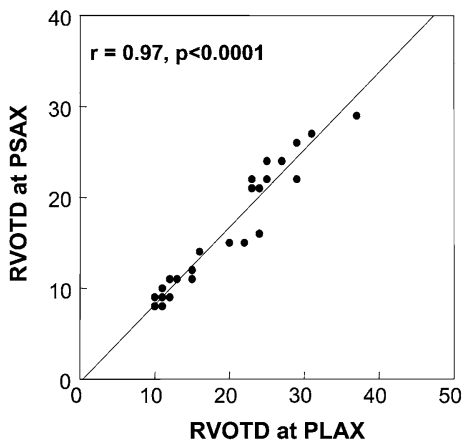

PVAD by 2DE PSAX and PLAX

(D)

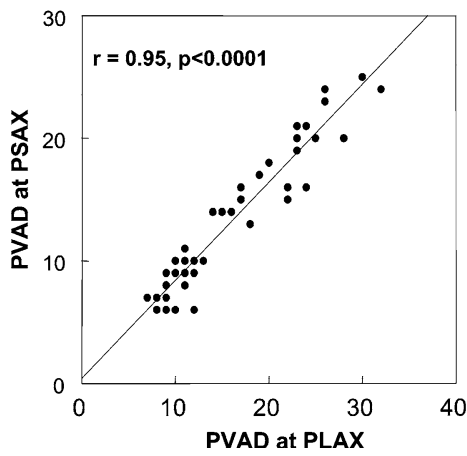

RVOTD by 2DE (PLAX) and by RT3DE RVOTD by 2DE (PSAX) and by RT3DE

(B)

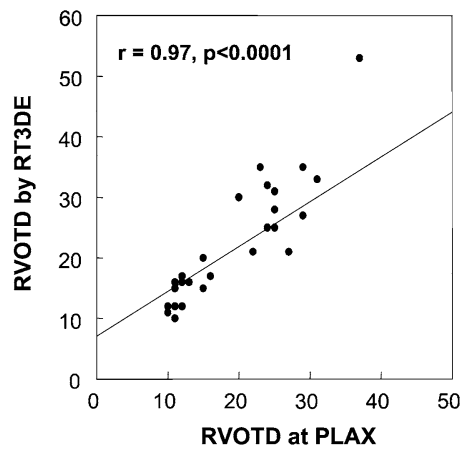

PVAD by 2DE (PLAX) \& by RT3DE
(C)

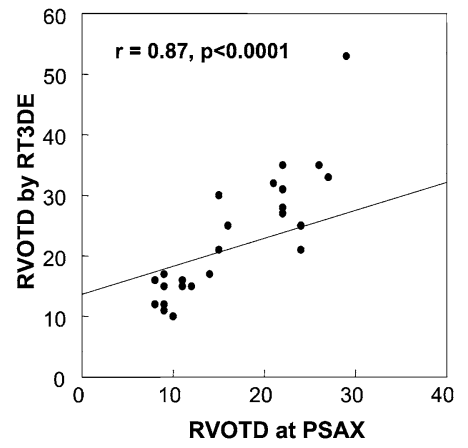

(E)

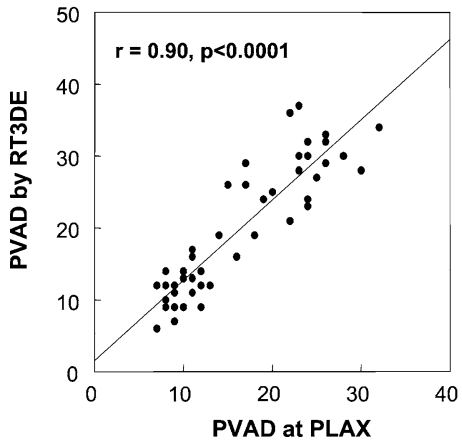

(F)

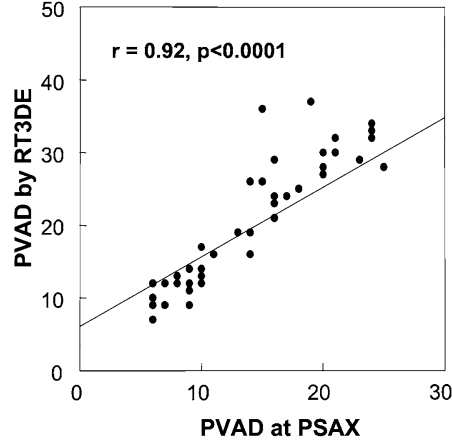

Fig. 2 (A) Correlation between RVOTD measured by 2DE in PLAX and PSAX, (B) Correlation between RVOTD measured by 2DE in PLAX and max RVOTD measured by RT3DE, (C) Correlation between RVOTD measured by $2 \mathrm{DE}$ in PSAX and max RVOTD measured by RT3DE, (D) Correlation between PVAD measured by 2DE in PLAX and PSAX, (E) Correlation between PVAD measured by 2DE in PLAX and that measured by RT3DE, and (F) Correlation between PVAD measured by $2 \mathrm{DE}$ in PSAX and that measured by RT3DE

Table 2 Measurements of 2DE and RT3DE

\begin{tabular}{|c|c|c|c|c|c|}
\hline & 2DE (PLAX) & 2DE (PSAX) & RT3DE & $P$-value* & $P$-value** \\
\hline RVOTD (mm) & $19.7 \pm 7.7$ & $16.4 \pm 6.8$ & $\begin{array}{l}22.2 \pm 10.0(\max ) \\
15.7 \pm 6.2(\min )\end{array}$ & 0.0001 & 0.001 \\
\hline PVAD (mm) & $16.4 \pm 7.3$ & $13.5 \pm 6.1$ & $19.4 \pm 9.1$ & 0.0001 & 0.0001 \\
\hline PPAD (mm) & $18.3 \pm 7.1$ & $18.1 \pm 7.1$ & $17.4 \pm 5.3$ & NS & NS \\
\hline DPAD (mm) & $19.3 \pm 9.1$ & $19.1 \pm 8.0$ & $18.6 \pm 6.4$ & NS & NS \\
\hline
\end{tabular}

Abbreviations: 2DE: 2-dimensional echocardiography, PLAX: parasternal long axis, PSAX: parasternal short axis, RT3DE: real-time 3-dimensional echocardiography, DPAD: distal pulmonary artery diameters, PPAD: proximal pulmonary artery diameter, PVAD: pulmonary valve annulus diameter, and right ventricular outflow tract (RVOT)

* $P$-value between $2 \mathrm{DE}$ measurements at both views (PLAX and PSAX)

**P-value between measurements of RT3DE and 2DE at PLAX

for RVOTD and PVAD. According to the Bland-Altman analysis of agreement, the mean differences were (mean difference: $-0.15 \pm 1.4$, agreement: $2.65-2.95 ; P=0.7$ ) (mean difference: - $0.57 \pm 1.90$, agreement: $3.33-4.37 ; P=0.09)$ (mean difference: $\quad 0.08 \pm 1.5$, agreement: $3.08-2.92$; 
Table 3 Scores for RT3DE visualization of RVOT, PVA, PV, PPA and DPA

\begin{tabular}{lccccc}
\hline Score & RVOT & PVA & PV & PPA & DPA \\
\hline Excellent (4) & $20(40 \%)$ & $44(88 \%)$ & $12(24 \%)$ & $29(58 \%)$ & $15(30 \%)$ \\
Sufficient (3) & $6(12 \%)$ & $4(8 \%)$ & $34(68 \%)$ & $10(20 \%)$ & $8(16 \%)$ \\
Inadequate (2) & $0(0 \%)$ & $0(0 \%)$ & $4(8 \%)$ & $0(0 \%)$ & $0(0 \%)$ \\
Missed (1) & $24(48 \%)$ & $2(4 \%)$ & $0(0 \%)$ & $11(22 \%)$ & $27(54 \%)$ \\
Median score & 2.4 & 3.8 & 3.1 & 3.1 & 2.2 \\
Mean score & $2.4 \pm 1.4$ & $3.8 \pm 0.6$ & $3.2 \pm 0.6$ & 3.5 & $2.2 \pm 1.4$ \\
\hline
\end{tabular}

Abbreviations: DPAD: distal pulmonary artery, PPAD: proximal pulmonary artery, PV: pulmonary valve, PVA: pulmonary valve annulus and RVOT: right ventricular outflow tract

Table 4 Value of RT3DE compared to 2DE for clinical assessment

\begin{tabular}{lll}
\hline & 2DE & RT3DE \\
\hline RVOT shape & - & ++ \\
RVOTA & - & ++ \\
RVOTD & + & ++ \\
PVAA & - & ++ \\
PVAD & + & ++ \\
PPAD & + & + \\
DPAD & + & + \\
\hline
\end{tabular}

$(-)$ : clinical assessment is not possible, $(+)$ : clinical assessment possible, and (++): increased clinical assessment

$P=0.7)$ (mean difference: $-0.40 \pm 1.90$, agreement: $3.40-4.20 ; P=0.1)$ for RVOT by $2 \mathrm{DE}$, PVAD by 2DE, RVOT by RT3DE, and PVAD by RT3DE, respectively (Figs. 3, 4).

\section{Discussion}

The study applied RT3DE for the assessment of PV and RVOT morphology and compared its measurements to those obtained with 2DE. This assessment could be obtained excellently or at least sufficiently in a higher percentage of patients. The main findings of our study are (1) RVOT shape was not circular but oval, (2) RVOTA and PVAA could be measured by RT3DE, and (3) RT3DE measurements of RVOTD and PVAD were higher than 2DE measurements. Assessment of PV and RVOT is of clinical importance because the understanding of normal morphology and function helps in detection of anomalies and selection of therapeutic strategies. For example, in pulmonary stenosis, therapeutic strategy is totally dependent on RVOT and PV morphology and function. The incidence of anomalies included within the term of pulmonary stenosis varies between $10.7 \%$ and $30 \%$ of all congenital heart defects [10, 11]. Despite this incidence, the pulmonary valve is the least studied valve. Its assessment is totally dependent on $2 \mathrm{DE}$, but images often less satisfactory in adolescents and adults [7]. The use of pulsed and continuous wave Doppler helps in accurate estimation of subvalvular and transvalvular pressure gradients for detection of pulmonary stenosis and selection for balloon valvuloplasty [12]. Several metods have attempted to accurately characterize PV morphology and detailed anatomy [13]. Kinelitze et al. [14] used bright blood cine magnetic resonance to visualize leaflets of the valve in 14 patients following Ross operation and he achieved good visualization with signal to noise ratio $7.5 \pm 2.2$ in patient group versus $9.8 \pm 3.0$ in normal control group. Berdajs et al. constructed a geometric model of the pulmonary root in 20 normals. By morphometric measurements of the distances between commissures and between intervalvular triangles, they described the asymmetrical structure of pulmonary root [15]. The current study shows that measurement of PVAD and RVOTD by 2DE according to the standard echocardiographic views leads to significant underestimation in comparison with RT3DE measurements. This could be explained by the fact that $2 \mathrm{DE}$ measures the distance from the basal attachment of one leaflet to the basal attachment of adjacent leaflet and these lines do not necessarily span the full diameter of RVOT but provide a tangential profile of the outlet [16]. 

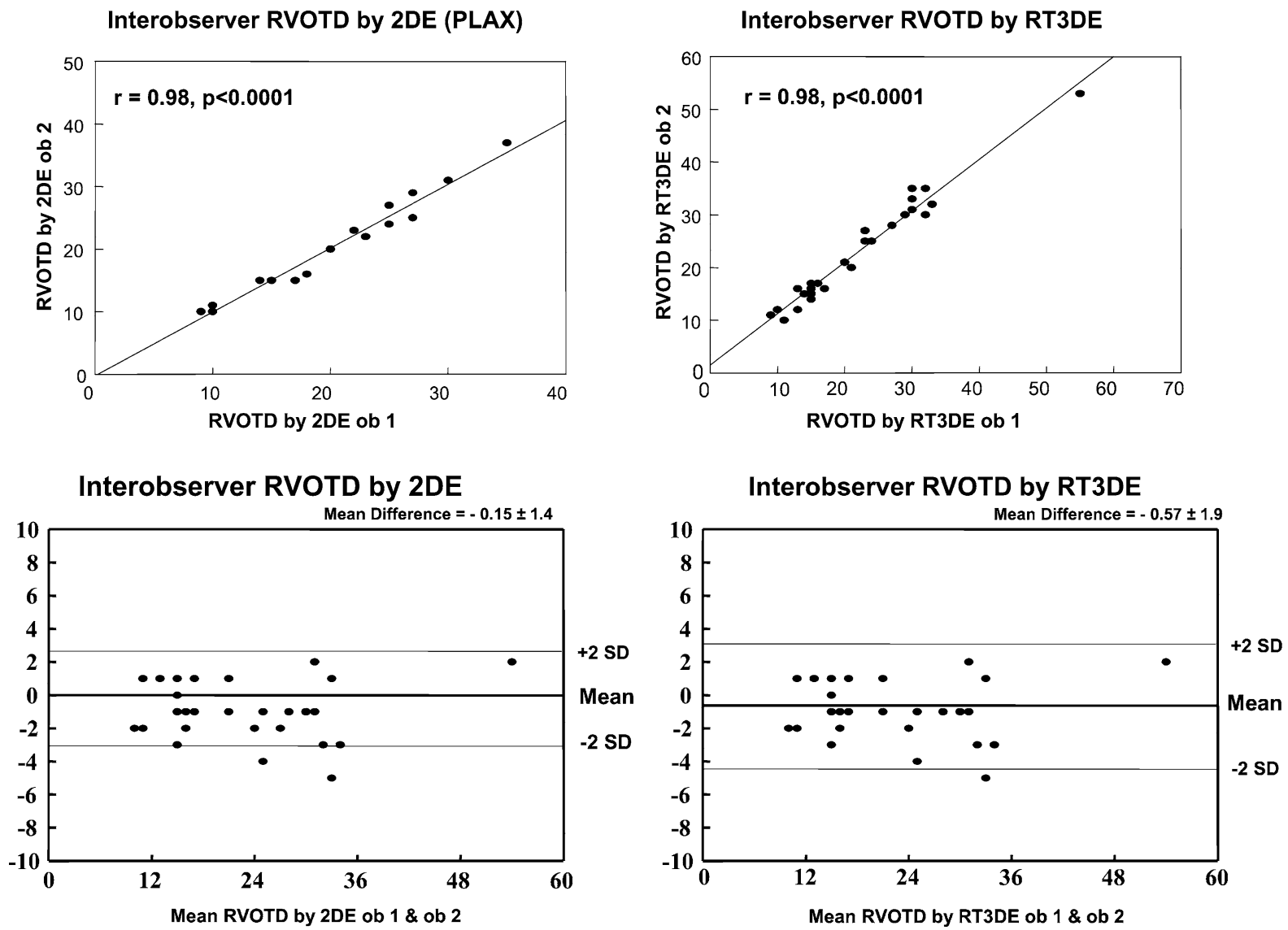

Fig. 3 Interobserver correlation and agreement according to Bland and Altman principle for measuring RVOTD by 2DE and RT3DE

Lines of RVOTD and PVAD measurements from PSAX view are perpendicular on the max RVOTD and PVAD by RT3DE. Thus, if 2DE is the only available method for PV and RVOT assessment, PLAX view should be used due to better visualization, and more alignment with RT3DE measurements of RVOTD and PVAD than PSAX. The study showed that RT3DE achieved excellent visualization of PVAA and calculation of its diameters in $88 \%$ of patients. In contrast, RVOT visualization and diameter measurement was not optimal and this may be due to tissue dropout and gain adjustment during acquisition. The PV visualization was sufficiently achieved in $92 \%$ of patients. The number, thickness and mobility of leaflets could be clearly visualized in $70 \%$ of patients. Commissures and direction of closure lines also could be visualized. Since calculation of PV area was not obtained well in considerable number of studied group and had wide interobserver variability, it was excluded from further analysis. This may be due to multiple levels at which area could be calculated as well as time points; no standardized method is available. Providing these data potentially constitute future application of RT3DE in a wider clinical routine and may enhance understanding valve morphology by providing accurate measurements necessary for selection of proper management (balloon valvuloplasty, surgical valvotomy, stented pulmonary valve or valve replacement). Another advantage of RT3DE application was visualization of PPA in $78 \%$ of patients and DPA in $46 \%$ of patients, which may help in visualization of supravalvular stenosis 
Interobserver PAD by 2DE (PLAX)

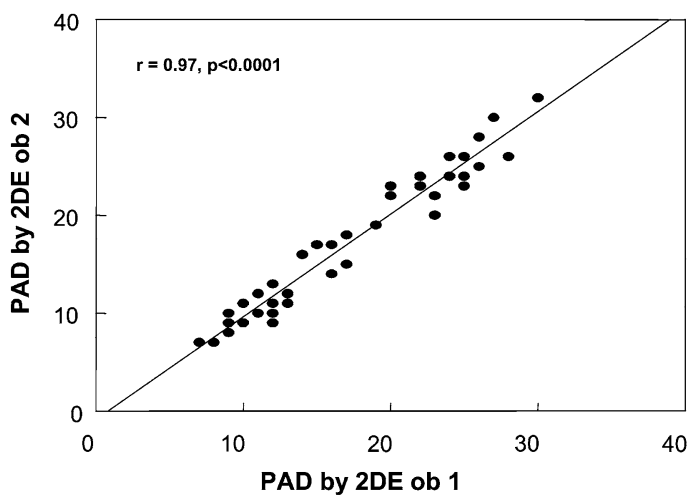

Interobserver PVAD by 2DE

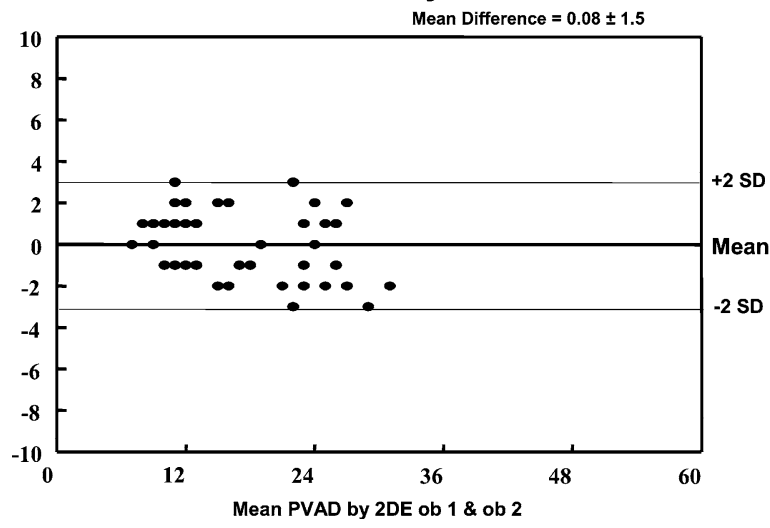

Interobserver PAD by RT3DE

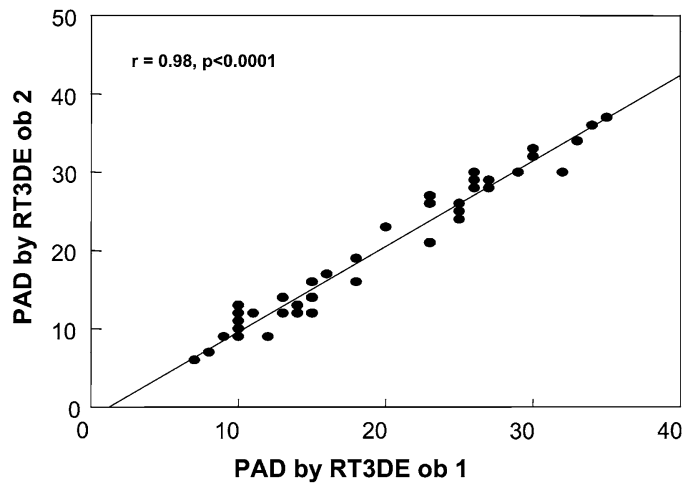

Interobserver PVAD by RT3DE

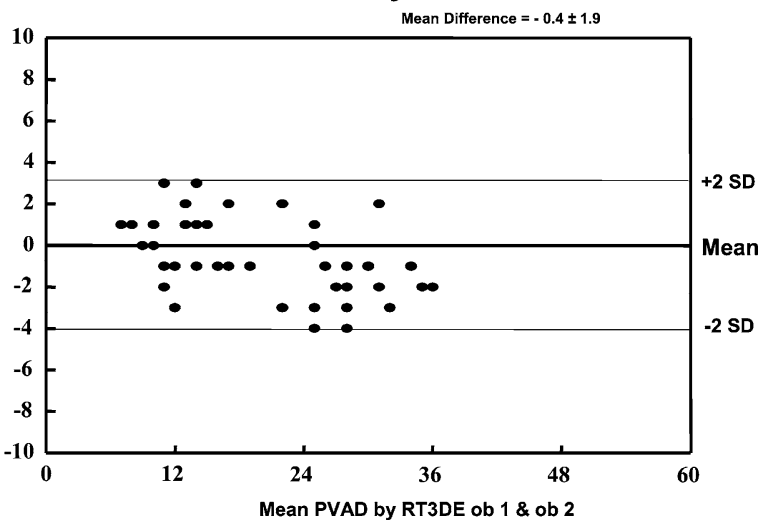

Fig. 4 Interobserver correlation and agreement according to Bland and Altman principle for measuring PVAD by 2DE and RT3DE

(by measuring luminal reduction and level(s) of stenosis), post-stenotic dilatation (by measuring $\%$ of luminal dilatation) or evaluation prior to surgery (shunts or correction).

\subsection{Limitation of study}

The study was conducted in a small number of patients characterized by heterogeneous congenital heart defects. The age of patients $32 \pm 9.5$ years affected the image quality of $2 \mathrm{DE}$ and RT3DE leading to significant difficulties in visualization of the RVOT. The data of the studied patients were not correlated with direct surgical visualization because a considerable number of patients were studied after corrective surgery or surgery was not the selected therapeutic strategy.

\subsection{Perspective}

The clinical value of RT3DE has to be validated in future studies by including selected group of patients with pulmonary stenosis with reference to intraoperative findings or MRI. For example a comparison of PV area by RT3DE at various levels to surgical findings could be helpful to develop a standardized approach to PV assessment.

\section{Conclusion}

RT3DE could help in clarification of detailed anatomy of RVOT and PV beyond the scope of established 2DE. A detailed assessment may provide a basis for proper selection of therapeutic strategy. 


\section{References}

1. Lang RM, Bierig M, Devereux RB, Flachskampf FA, Foster E, Pellikka PA, Picard MH, Roman MJ, Seward J, Shanewise JS, Solomon SD, Spencer KT, Sutton MS, Stewart WJ (2005) Recommendations for chamber quantification: a report from the American Society of Echocardiography's Guidelines and Standards Committee and the Chamber Quantification Writing Group, developed in conjunction with the European Association of Echocardiography, a branch of the European Society of Cardiology. J Am Soc Echocardiogr 18:1440-63

2. King MEE (2002) Echocardiographic evaluation of the adult with unoperated congenital heart disease. In: Otto C (ed) The practice of clinical echocardiography. WB Saunders, pp 868-899

3. Stumper O, Witsenburg M, Sutherland GR, CrommeDijkhuis A, Godman MJ, Hess J (1991) Transesophageal echocardiographic monitoring of interventional cardiac catheterization in children. J Am Coll Cardiol 18:1506-14

4. Vick GW 3rd, Rokey R, Huhta JC, Mulvagh SL, Johnston DL (1990) Nuclear magnetic resonance imaging of the pulmonary arteries, subpulmonary region, and aorticopulmonary shunts: a comparative study with two-dimensional echocardiography and angiography. Am Heart J 119:1103-10

5. McAleer E, Kort S, Rosenzweig BP, Katz ES, Tunick PA, Phoon CK, Kronzon I (2001) Unusual echocardiographic views of bicuspid and tricuspid pulmonic valves. J Am Soc Echocardiogr 14:1036-8

6. Vogel M, Ho SY, Lincoln C, Yacoub MH, Anderson RH (1995) Three-dimensional echocardiography can simulate intraoperative visualization of congenitally malformed hearts. Ann Thorac Surg 60:1282-8
7. Foale R, Nihoyannopoulos P, McKenna W, Kleinebenne A, Nadazdin A, Rowland E, Smith G (1986) Echocardiographic measurement of the normal adult right ventricle. Br Heart J 56:33-44

8. Byrt T (1996) How good is that agreement?. Epidemiology 7:561

9. Bland JM, Altman DG (1986) Statistical methods for assessing agreement between two methods of clinical measurement. Lancet 1:307-10

10. Hoffman JI (1995) Incidence of congenital heart disease: I. Postnatal incidence. Pediatr Cardiol 16:103-13

11. Rocchini APE (1995) Pulmonary stenosis. In: Emmanouilides GCRT, Allen HD, Gutgesell HP (eds) Moss and Adams' Heart disease in infants, children, and adolescents. William \& Wilkins, Baltimore, pp 930-962

12. Mulhern KM, Skorton DJ (1993) Echocardiographic evaluation of isolated pulmonary valve disease in adolescents and adults. Echocardiography 10:533-43

13. Nascimento R, Campelo M, Maciel J, Lourenco A, Carneiro M, Cunha D, Van-Zeller P (1993) [Echocardiographic evaluation of pulmonary valve stenosis for valvuloplasty in children and adults]. Rev Port Cardiol 12:141-50

14. Kivelitz DE, Dohmen PM, Lembcke A, Kroencke TJ, Klingebiel R, Hamm B, Konertz W, Taupitz M (2003) Visualization of the pulmonary valve using cine MR imaging. Acta Radiol 44:172-6

15. Berdajs D, Lajos P, Zund G, Turina M (2005) Geometrical model of the pulmonary root. J Heart Valve Dis 14:257-60

16. Martinez RM, Anderson RH (2005) Echocardiographic features of the morphologically right ventriculo-arterial junction. Cardiol Young 15(Suppl1):17-26 